\title{
Molecular Docking Interaction of Mycobacterium Tuberculosis LipB Enzyme with Isoniazid, Pyrazinamide and a Structurally Altered Drug 2, 6 Dimethoxyisonicotinohydrazide
}

\author{
Muthuraman Namasivayam, ${ }^{1,}$, Suresh Ramraj Subashchandrabose ${ }^{2}$ \\ ${ }^{1}$ Center of Advance Study in Crystallography and Biophysics, University of Madras, Chennai, India \\ ${ }^{2}$ Global Centre for Environmental Risk Assessment and Remediation Faculty of Science and Information Technology, The University of New \\ Castle, Newcastle, Australia
}

Email address:

muthuraman87@gmail.com (M. Namasivayam), suresh.subashchandrabose@newcastle.edu.au (S. R. Subashchandrabose)

\section{To cite this article:}

Muthuraman Namasivayam, Suresh Ramraj Subashchandrabose. Molecular Docking Interaction of Mycobacterium Tuberculosis LipB Enzyme with Isoniazid, Pyrazinamide and a Structurally Altered Drug 2, 6 Dimethoxyisonicotinohydrazide. Computational Biology and Bioinformatics. Vol. 3, No. 4, 2015, pp. 45-51. doi: 10.11648/j.cbb.20150304.11

\begin{abstract}
Tuberculosis is an infectious airborne disease caused by a bacterial infection that affects the lungs and other parts of the body. Vaccination against tuberculosis is available but proved to be unsuccessful against emerging multi drug and extensive drug resistant bacterial strains. This in turn raises the pressure to speed up the research on developing new and more efficient anti-tuberculosis drugs. Lipoate biosynthesis protein B (LipB) is found to play vital role in the lipoylation process in Mycobacterium tuberculosis and thus making it a very promising drug target. The existing first line drugs such as Isoniazid, Pyrazinamide and Rifampicin etc shows only profound binding affinity with this target protein. Therefore, new or modified drugs with better docking approach that exhibit a closer and stronger binding affinity is essential. This current study opens up a novel approach towards anti-tuberculosis agents by determining drugs that share similar structures with some of the best available first line drug and also happen to possess better binding affinity. In this article, a computational method by which, pristine as well certain first line and structurally modified drugs were docked with the LipB protein target; where, structurally modified 2, 6 Dimethoxyisonicotinohydrazide show superior target docking.
\end{abstract}

Keywords: Mycobacterium Tuberculosis, Multi-drug Resistant Tuberculosis, Anti-tuberculosis Drugs, LipB, Isoniazid, Lipoylation, Molecular Docking, Drug Design, 2,6 Dimethoxyisonicotinohydrazide

\section{Introduction}

Mycobacterium Tuberculosis is a pathogenic bacterial species responsible for causing tuberculosis (TB). It mainly affects the lungs and extensively capable of affecting other parts of the body such as spine, kidney, and even brain unless timely treatment is provided. TB remains one of the most prevalent infections resulting in a worldwide death of 1.4 million and an estimated new case of about 8.7 million. The typical symptoms of TB are chronic cough with blood tinged sputum, fever, weight loss, night sweats and if untreated resulting in death ultimately (Ismail, 2004; Cole et al, 2004).

Although there are drug like isoniazid, rifampicin and ethambutol available as a cure for $\mathrm{TB}$, emergence of multidrug resistant (MDR) and extensively drug resistant (XDR) tuberculosis that resist current drug calls poses a big challenge towards successful treatment of tuberculosis (Gyanu, 2011)). This in turn drives the current research to a faster pace in order to develop new therapeutics against diverse strains of M. Tuberculosis (WHO, 2010; Davies, 1999). There are several enzymes that participate in the pathogenicity vital metabolic process like growth of the bacterium and one among them is Lipoate biosynthesis protein B (LipB).

LipB is the enzyme that is involved in lipoylation; it catalyses the transfer of endogenous octanoic acid to lipoyl domains by way of a thioester bond to the 4'- 
phosphopanthetheine cofactor of the acyl carrier protein (ACP).The octanoyl derivatives are then converted into lipoyl derivatives by another enzyme known as lipoyl synthase (Lip A) thus finds a way to serve the need of the bacteria for exogenous lipoic acids [6]. Thus it acts as the necessary protein involved in activating the cellular machines that drives the bacterium's metabolism. Moreover, an up regulated level of Lip B has been observed in patients infected by multidrug resistant forms of M.Tuberculosis and there happens to be no other known back-up mechanism that can take over LipB's role in the metabolism of TB (Zhao et al, 2005; Quin and John, 2010). Furthermore, generating a mutant lacking LipB gene resulted in a less virulent and defective bacterium confirming the theory of LipB's importance in the growth of M. Tuberculosis thus making it a viable target for developing anti-TB drugs (Quinjun Ma et al, 2006).

Drug screening against TB has been done for years now, showing success at varying rates but in the recent times there had been severe limitations in wet lab approach due to the requirement of high level scientific expertise with costly laboratory settings for handling the pathogen like $\mathrm{M}$. Tuberculosis. However, development in computation methods in the recent times have taken some interesting turns. In the past, drugs have been discovered through identification of active ingredients from traditional remedies or by unexpected discovery. This has not provided with an opportunity to understand and control diseases and infection at the molecular and physiological level (Kitchen et al, 2004). Whereas, with the help of computational methods basic knowledge regarding the ligand and receptor can be acquired beforehand and also are very much capable to manipulate and target specific entities. These actions are very much useful in molecular docking, structure and ligand based drug screening and many more.

This dry lab research also helps to evaluate the binding affinity between a ligand and its receptor thus helping to prioritize drug candidates for screening in a wet lab approach. In order to experiment a virtual screening process, a 3D structural data of the protein and the receptor ligand that has been constructed through laboratory procedures like NMR spectroscopy, high energy synchrotron radiation beamlines, and X-ray crystallography is suffice.

Protein ligand docking is a computational method developed to interpret the preferred orientation between two molecules in order for them to form a stable complex; indeed it plays a key role in computer assisted drug design. Success rate of docking can be predicted using a scoring function that ranks candidate docking correctly by predicting the strength of association or binding affinity between the two molecules. The scoring functions are based on the molecular mechanical force fields that estimate the required energy of the pose (orientation) (Kitchen et al, 2004). The most important part of docking is setting up of input structure for the docking and is just as important as the docking itself. There are currently several databases online that helps by providing with all the information, one need about different protein molecules and diverse chemicals and drugs.

In the present study, the 3-D structure of LipB is constructed using the FASTA sequence obtained from NCBI database. Further, a structurally modified drug, showing similarity to an existing drug is obtained from Enhanced NCI database and was allowed to dock with the acquired LipB structure. The overall binding energy was used as the score to compare the docking interaction, eventually analysing the stable complex. The studies presented in this manuscript will be useful to design drug that exhibits high activity against multidrug or extensive drug resistant tuberculosis.

\section{Materials and Methods}

\subsection{Structural Data of LipB}

National Centre for Biotechnology Information (NCBI) is a comprehensive website for biologists, that include biology related databases and necessary tools for analysis. The query sequence of LipB (Gene ID: 887626) is retrieved from the database in FASTA format and using Basic Local Alignment Search Tool (BLAST), a similarity search is performed in order to find the template sequence of LipB and then using Protein data Bank (PDB), the template structure is constructed from the template sequence (Altschul et al, 1997).

Swisspdb viewer is a user friendly application, which mainly helps to analyse several protein sequence at the same time and capable of superimposing proteins in order to deduce the structural alignment. SWISS-Model is a fully automated program that can be accessed directly from Swisspdb viewer and this program makes the Protein modelling accessible worldwide. Working with these programs greatly reduces the amount of work necessary to generate models, as with the help of these programs it is possible to thread a protein primary sequence onto a $3 \mathrm{D}$ template and acquire an immediate feedback of how well the threaded protein will be accepted by the reference structure. Confirming the stability of the protein structure is very important, Structural Analysis and Verification Server (SAVES) tool can be used for this process, as it confirms the stability of the protein structure by analysing the residue-by-residue geometry and overall structural geometry (Arnold et al, 2006).

\subsection{Enhanced NCI Database}

Enhanced NCI database is an online tool that is capable of enabling a wide scale search for drugs and them aids to find a drug with high structural similarity as that of the chemical structure of the existing drug. Further it provides details regarding their molecular weight, chemical structure, 3D structure and chemical composition.

The drug that has the highest structural similarity with an existing drug was selected to dock with the LipB protein enzyme. 


\subsection{Computer Aided Molecular Docking}

Lip B structure acquired from SWISS model, after confirming for the stability was allowed to dock with acquired drug from NCI database; the calculated binding energy was used as the scoring function and was employed as the baseline comparison between the new identified drug and existing drug (Dominguez et al, 2003).

$H E X$, an interactive docking tool that can be used to dock the proteins and the ligands, $H E X$ can calculate the binding energy required for that specific protein-ligand docking and it can also superpose two molecules with only knowledge of their 3D structure (Hex User Manual). The binding energy result is normally negative, stating that a better binding affinity is established from a highest negative result thus illustrating that low (negative) energy indicates a stable system and thus a likely interaction (Ritchie, 2003).

Normally, two approaches are popular with the molecular docking community. Shape complementary method uses a matching technique that treats the protein and the ligand as complementary surfaces. Whereas, the simulation process imitates an actual docking process where both the protein and ligand's pair wise interaction energy is calculated.

Shape complementary method describes protein and ligand as a set of features that amounts to shape matching description that may help finding the complementary pose of docking the two molecules. A quick scan through several thousand ligands in a matter of seconds is actually possible, to figure out whether they can bind at the protein's active site. Both the LipB protein and the drug were oriented in order for a best fit and the energy required is estimated.

In the Simulation method, the ligand that is separated from the protein by a physical distance finds its position into the protein's active site after a certain number of "moves" in its conformational space, such as translations, rotations and even internal changes into ligand's structure including torsion angle rotation. The total energy of the system is calculated after every move, the interaction between LipB and the drug by this method is very much closer to what happens in reality and thus the position at which the lowest energy is recorded is considered to be the best fit (Taylor and Burnett, 2000; Smith and Sternberg, 2002)

\section{Results and Discussion}

The query sequence for $1.08-\AA 3 \mathrm{D}$ structure Lipoate biosynthesis protein $\mathrm{B}$ published in protein data bank(Junie et al, 2013)was retrieved from the NCBI database in FASTA format as shown in seq no. 1 ,

"MTGSIRSKLSAIDVRQLGTVDYRTAWQLQRELADA RVAGGADTLLLLEHPAVYTAGRRTETHERPIDGTPVVD TDRGGKITWHGPGQLVGYPIIGLAEPLDVVNYVRRLE ESLIQVCADLGLHAGRVDGRSGVWLPGRPARKVAAIG VRVSRATTLHGFALNCDCDLAAFTAIVPCGISDAAVTS LSAELGRTVTVDEVRATVAAAVCAALDGVLPVGDRVP SHAVPSPL"

Seq. No. 01: Query sequence of LipB
This query sequence represents the amino acid codes of the LipB protein and BLAST is performed in order to find regions of similarity between this sequence and other sequences.

A pair wise alignment is done to find the similarity between query and the template sequence as shown below. In the total of 92 blast hits with varied alignment values, $>90 \%$ similarity of 230 amino acid codes has been observed in the different strains of M.Tuberculosis and an identity of $100 \%$ has been found in M.Tuberculosis [H37Rv]. Thus, makes LipB a highly valuable target for anti-tuberculosis drugs. Moreover, Qingjun Ma et al demonstrated that LipB possess some promising target properties in terms of its altered pattern during disease and peculiar structural property revealing a large active site for its substrate (Qingjun Ma et al, 2006).

\begin{tabular}{lll}
\hline Identities & Positives & Gaps \\
\hline $230 / 230(100 \%)$ & $230 / 230(100 \%)$ & $0 / 230(0 \%)$ \\
\hline
\end{tabular}

Query 1

MTGSIRSKLSAIDVRQLGTVDYRTAWQLQRELADAR

VAGGADTLLLLEHPAVYTAGRRTE 60

MTGSIRSKLSAIDVRQLGTVDYRTAWQLQRELADAR VAGGADTLLLLEHPAVYTAGRRTE

Sbjet 1

MTGSIRSKLSAIDVRQLGTVDYRTAWQLQRELADAR VAGGADTLLLLEHPAVYTAGRRTE 60

Query 61

THERPIDGTPVVDTDRGGKITWHGPGQLVGYPIIGL

AEPLDVVNYVRRLEESLIQVCADL 120

THERPIDGTPVVDTDRGGKITWHGPGQLVGYPIIGL AEPLDVVNYVRRLEESLIQVCADL

Sbjct 61

THERPIDGTPVVDTDRGGKITWHGPGQLVGYPIIGL AEPLDVVNYVRRLEESLIQVCADL 120

Query 121

GLHAGRVDGRSGVWLPGRPARKVAAIGVRVSRATT

LHGFALNCDCDLAAFTAIVPCGISD 180

GLHAGRVDGRSGVWLPGRPARKVAAIGVRVSRATT

LHGFALNCDCDLAAFTAIVPCGISD

Sbjet 121

GLHAGRVDGRSGVWLPGRPARKVAAIGVRVSRATT

LHGFALNCDCDLAAFTAIVPCGISD 180

Query 181

AAVTSLSAELGRTVTVDEVRATVAAAVCAALDGVLP VGDRVPSHAVPSPL 230

AAVTSLSAELGRTVTVDEVRATVAAAVCAALDGVLP VGDRVPSHAVPSPL

Sbjct 181

AAVTSLSAELGRTVTVDEVRATVAAAVCAALDGVLP VGDRVPSHAVPSPL 230

Sequence similarity between LipB (query) and M.Tuberculosis (template) [H37Rv] 


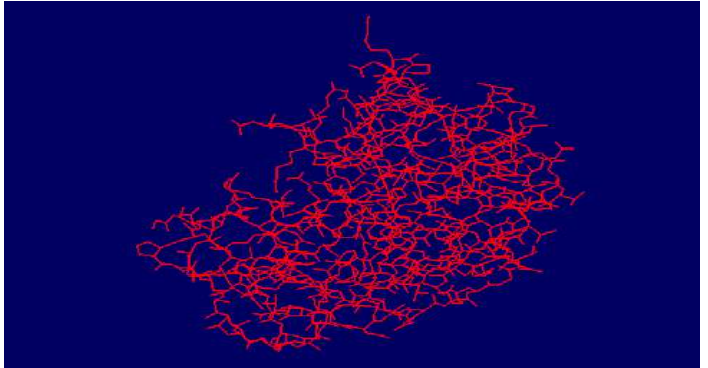

Fig. No.01. Modelled structure of LipB.

Modelling is done using Swiss pdb viewer, primarily the raw sequence obtained from NCBI is loaded and then the PDB structure of the template is loaded. The RAW sequence is fit to the template using MAGIC FIT, and finally the modelled structure is obtained from SWISS model, as shown in the figure No. 01.

First line drugs available in the market that treats M.Tuberculosis are Isoniazid, Ethambutol, Pyrazinamide and Rifampicin. Among them both Isoniazid and Pyrazinamide happens to be very effective in serious cases of TB. These drugs were allowed to dock with the modelled template structure obtained from SWISS model using HEX tool as shown in figure 02 and 03 . The scoring function is by mainly estimating the binding energy at which the drugs dock and the lowest binding energy happens to provide a more stable system.

Table No. 01. Bond length of the specific amino acid residues of LipB protein that dock with Isoniazid drug.

\begin{tabular}{llll}
\hline $\begin{array}{l}\text { Amino acid } \\
\text { residue }\end{array}$ & Bond Length & $\begin{array}{l}\text { Amino acid } \\
\text { residue }\end{array}$ & Bond Length \\
\hline$T R P 12$ & $2.71 \AA$ & $A L A 90$ & $2.11 \AA$ \\
$L Y S 72$ & $2.97 \AA$ & $A S N 92$ & $4.72 \AA$ \\
$V A L 73$ & $1.94 \AA$ & $P H E 100$ & $1.12 \AA$ \\
$A L A 74$ & $1.23 \AA$ & $A L A 112$ & $6.79 \AA$ \\
$A L A 75$ & $3.49 \AA$ & $V A L 113$ & $2.74 \AA$ \\
\hline
\end{tabular}

Isoniazid:

E Total: -138.44

E Shape: -138.44

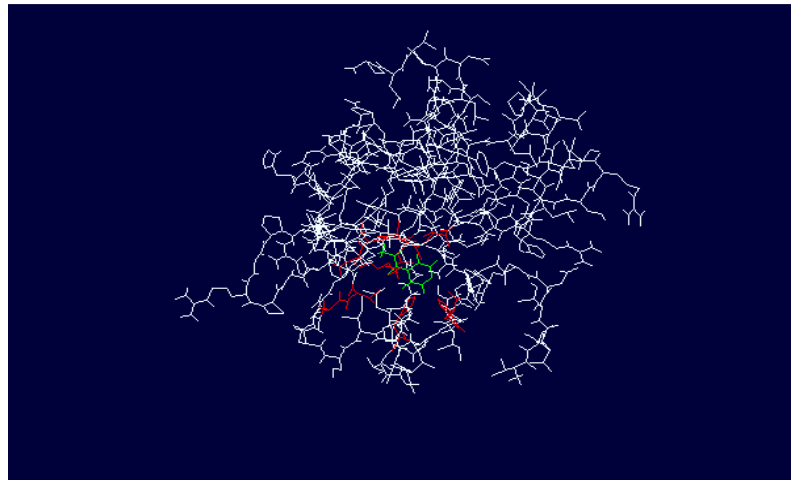

LipB (white), Docked amino acids (Red) and Isoniazid drug (Green).

Pyrazinamide:

E Total: -116.00

E Shape: -116.00

Fig. No. 02. Docking interaction between LipB and Isoniazid.
Table No. 02. Bond length of the specific amino acid residues of LipB protein that dock with Pyrazinamide drug.

\begin{tabular}{llll}
\hline $\begin{array}{l}\text { Amino acid } \\
\text { residue }\end{array}$ & Bond Length & $\begin{array}{l}\text { Amino acid } \\
\text { residue }\end{array}$ & Bond Length \\
\hline LEU 97 & $4.59 \AA$ & PHE 100 & $5.06 \AA$ \\
THR 101 & $4.59 \AA$ & PRO 105 & $4.89 \AA$ \\
ILE 108 & $3.90 \AA$ & SER 109 & $3.87 \AA$ \\
ASP 110 & $5.91 \AA$ & ALA 111 & $3.24 \AA$ \\
$A L A 112$ & $3.08 \AA$ & VAL 113 & $4.13 \AA$ \\
\hline
\end{tabular}

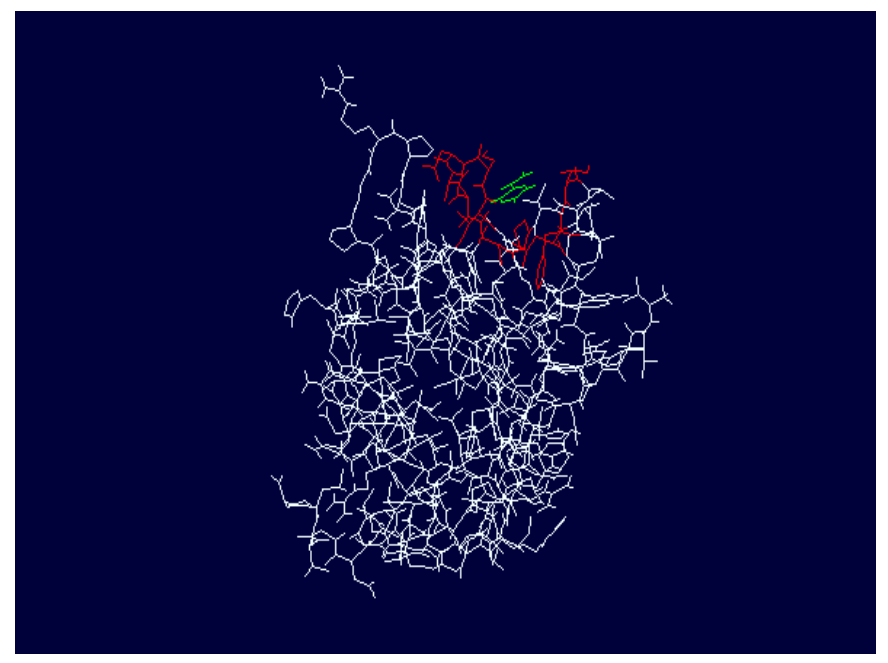

LipB (white), Docked amino acids (Red) and Pyrazinamide drug (Green)

Fig. No. 03. Docking interaction between LipB and Pyrazinamide.

Isoniazid and Pyrazinamide are two dissimilar drugs both structurally and compositionally, thus binds with the receptor protein at totally different binding sites in order to obtain best possible stable complex. The amino acids in the protein with which the drug docks shows and confirms that Isoniazid docks completely at different positions than Pyrazinamide. Binding energy (E-Total and E-Shape) in both these cases are recorded to be negative thus confirming that a good binding affinity has taken place between the protein and the drugs, but a better result is recorded for the interaction between Isoniazid and the LipB protein as comparatively they happened to require the lowest binding energy in order to dock. The total energy of the system (E-total) and the shape only energy (E-shape) showing similar reading illustrates that, the molecular docking in both the approach has happened at the best possible position.

Secondly, According to the bond length expressed in Angstrom units, isoniazid is capable for binding closer to the template structure than Pyrazinamide thus requiring low binding energy. Bhagat et al performed a study on target proteins for M.Tuberculosis, computed energy levels using HEX docking program (Bhagat et al, 2014). The result reported was very much similar to the energy levels we obtained for Isoniazid and Pyrazinamide drug against LipB protein target. Thus, confirming that Isoniazid drug forms a more stable complex than Pyrazinamide. 
Table No. 03. Energy comparison of Isoniazid and Pyrazinamide.

\begin{tabular}{lll}
\hline & Isoniazid & Pyrazinamide \\
\hline Total Energy of the System(E-total) & -138.44 & -116.00 \\
Energy of Shape only approach (E-shape) & -138.44 & -116.00 \\
\hline
\end{tabular}

The mechanism by which Isoniazid reacts is that it acts as a prodrug and is triggered by catalyse peroxidise enzyme called KatG present in M. Tuberculosis (Suarez et al, 2009). $\mathrm{KatG}$, presumed to couple isonicotinoyl radical with NADH forming an isoniazid-NADH complex. This complex binds tightly to an enoyl acyl carrier protein reductase known as InhA, which has a key role in the production of mycolic acid. Isoniazid-NADH complex inhibits InhA, thereby blocks the production of mycolic acids, eventually affecting the structural component of Mycobacterium cell wall (Cade et al, 2010).

The outcome of the docking results had allowed the development of a novel drug by altering the structure of Isoniazid with the help of enhanced NCI database. The database provides with the structure of all existing drugs and can also provide structural information of drugs with similar structures as of the existing drug. Ligand with high structure similarity was chosen. The reason being, this docking mechanism is mainly a method that predicts the preferred orientation between two molecules and analyses the amount of energy required for these molecules to form a stable complex (Brooijmans and Kuntz, 2003). So, they are expected to dock with the template structure in a binding affinity close to the original drug (Cheng et al, 2012). The advantage of a structurally similar drug would be that, the mechanism by which the drug reacts with the template structure could very much be similar to that of the original drug. Thus, a beforehand understanding of the novel drug and its way of reaction could be possible. So, a structurally similar ligand is a more appropriate option than a compositionally similar ligand.

The drug that has a fairly high structural similarity with the Isoniazid is 2, 6 Dimethoxyisonicotinohydrazide.

Structure of Isoniazid:

Chemical formula: $\mathrm{C}_{6} \mathrm{H}_{7} \mathrm{~N}_{3} \mathrm{O}$

Composition: C 52.55\% H 5.14\% N 30.64\% O 11.67\%

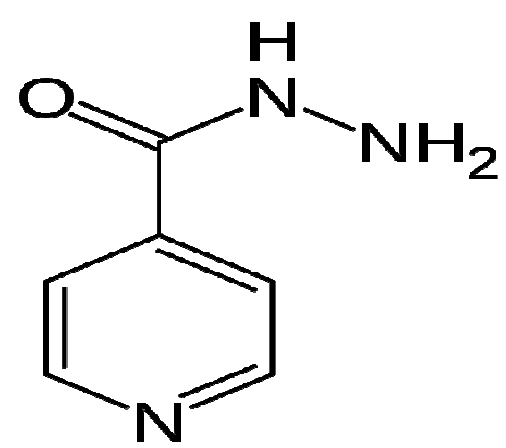

Structure of 2, 6 Dimethoxyisonicotinohydrazide:

Chemical formula: $\mathrm{C}_{8} \mathrm{H}_{11} \mathrm{~N}_{3} \mathrm{O}_{3}$

Composition: C 48.73\% H 5.62\% N 21.31\% O 24.34\%<smiles>COc1cc(C(=O)NN)cc(OC)n1</smiles>

The structure obtained was allowed to dock with the LipB protein; the docking interaction between them is shown in figure 4 and 5

2, 6 Dimethoxyisonicotinohydrazide:

E Total: -188.06

E Shape: -188.06

Table No. 04. Bond length of the specific amino acid residues of LipB protein that dock with Pyrazinamide drug.

\begin{tabular}{llll}
\hline $\begin{array}{l}\text { Amino acid } \\
\text { residue }\end{array}$ & Bond Length & $\begin{array}{l}\text { Amino acid } \\
\text { residue }\end{array}$ & Bond Length \\
\hline$A R G 6$ & $3.93 \AA$ & $A L A 204$ & $2.74 \AA$ \\
SEL 10 & $3.03 \AA$ & $A L A 205$ & $2.92 \AA$ \\
$A L A 11$ & $3.66 \AA$ & $C Y S 208$ & $2.30 \AA$ \\
$V A L 14$ & $3.32 \AA$ & $A L A 209$ & $4.01 \AA$ \\
$G L N 16$ & $4.72 \AA$ & $V A L 214$ & $2.69 \AA$ \\
\hline
\end{tabular}

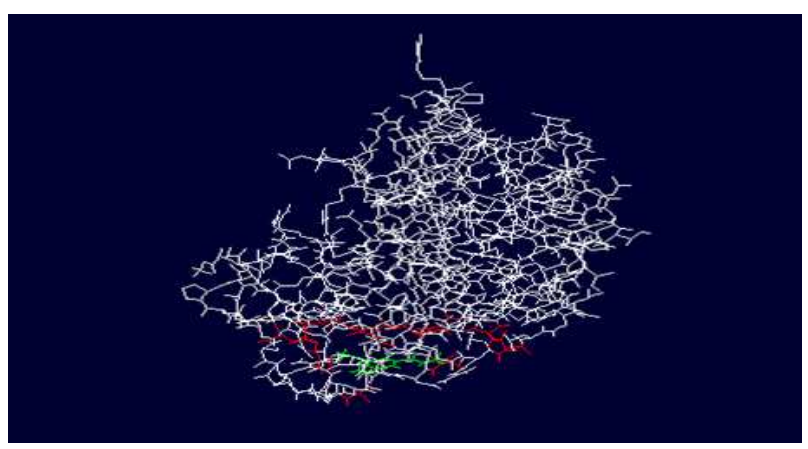

LipB (white), Docked amino acids (Red) and 2, 6 Dimethoxyisonicotinohydrazide drug (Green)

Fig. No. 04. Docking interaction between LipB and 2, 6 Dimethoxyisonicotinihydrazide.

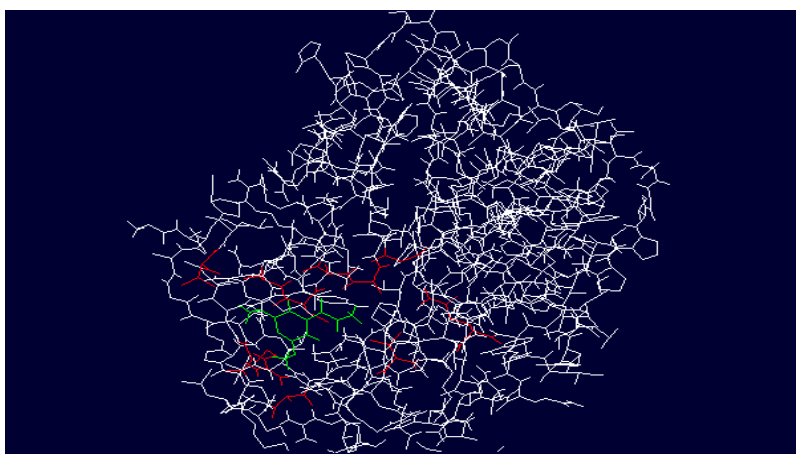

LipB (white), Docked amino acids (Red) and 2, 6

Dimethoxyisonicotinohydrazide drug (Green)

Fig. No. 05. Docking interaction (closer look) between LipB and 2, 6 Dimethoxyisonicotinihydrazide. 
The structurally similar drug Isoniazid and 2, 6 dimethoxyisonicotinohydrazide is observed to dock with the receptor protein at a different binding site. The reason being, even though both the drugs share almost similar structures, a slight structural modification in them is enough to create a difference in their binding affinity (Pereira de Jésus-Tran K et al, 2006). Moreover, binding between drug and receptor proteins depends mainly on the strength of interaction between them (Kastritis P.L et al, 2012). According to Induced Fit Theory, the receptor protein alters the conformation of its binding sites with respect to the structure of the ligands in order to produce a more stable drug-receptor complex. In the result observed, the binding sites for all three drugs were different and thus exhibit different binding affinity. From the observed docking mechanism, 2, 6 Dimethoxyisonicotinohydrazide and LipB (receptor protein), are capable of forming a stable complex at a binding energy much lower than Isoniazid i.e. the total energy (E-Total) of the system and the energy of the shape only (E-shape) approach both resulted to be comparatively lower than Isoniazid.

Table No. 05. Energy comparison of 2, 6 Dimethoxyisonicotinohydrazide and Isoniazid.

\begin{tabular}{|c|c|c|}
\hline & Isoniazid & 2, 6 Dimethoxyisonicotinohydrazide \\
\hline $\begin{array}{l}\text { Total Energy of the } \\
\text { System(E-total) }\end{array}$ & -138.44 & -188.06 \\
\hline $\begin{array}{l}\text { Energy of Shape } \\
\text { only approach (E- } \\
\text { shape) }\end{array}$ & -138.44 & -188.06 \\
\hline
\end{tabular}

\section{Conclusion}

Achieving a stable system is important when it comes to drug target protein docking and it can be attained by forming a better affinity between the protein and the drug. The amount of energy required by the drug in order to dock with the protein complex plays an important role. The lower the energy required is better for docking, as they are by theory forms a more stable system. The article illustrates that LipB as by design has a very unreplaceable role in the bacterium's metabolism succeeds as a viable target against multi drug resistant tuberculosis. The docking interaction between LipB and certain currently available drugs shows successful bonding but not promising enough. The structurally altered drug 2, 6 Dimethoxyisonicotinohydrazide, not only shows successful bonding but also shows a better affinity. The binding energy comparison analysis proves that 2,6 Dimethoxyisonicotinohydrazide needs much lesser binding energy to dock with LipB protein better than Isoniazid and other drugs, forming a more stable system. This likely makes it a more feasible agent in cases of anti-tuberculosis drugs.

\section{Appendix}

Internet tools used:

A. National Centre for Biotechnology Information

B. Protein Data Bank
C. SWISS-MODEL: Protein structure homologymodelling server

$D$. Enhanced NCI database

E. HEX: Protein docking and molecular superposition program

\section{Reference}

[1] Abad-Zapatero C. and Metz J.T. (2005) "Ligand efficiency indices as guideposts for drug discovery” Drug Discov. Today, 10:464-9.

[2] Altschul, S. F, Madden T.L, Schäffer A.A, Zhang J, Zhang Z, Miller W, and Lipman DJ. (1997) "Gapped BLAST and PSIBLAST: a new generation of protein database search programs." Nucleic Acids Res 25(17): 3389-402.

[3] Arnold, K., Bordoli L, Kopp J, and Schwede T (2006) "The SWISS-MODEL workspace: a web-based environment for protein structure homology modelling." Bioinformatics 22(2): 195-201.

[4] Brooijmans, N, Kuntz, I.D. (2003) "Molecular recognition and docking algorithms" Annu. Rev. Biophys.Biomol. Struct., $32,335-373$.

[5] C. B. Bhagat, S. K. Tank, P. R. Dudhagara N. D. Trivedi and U. N. Trivedi (2014) "In silico Study of Target Proteins for Mycobacterium tuberculosis" American Journal of Phytomedicine and Clinical Therapeutics 2, 3, 455-462.

[6] Cade C.E, Dlouhy A.C, Medzihradszky K.F, Salas-Castillo S.P, and Ghiladi R.A (2010) "Isoniazid-resistance conferring mutations in Mycobacterium tuberculosis KatG: catalase, peroxidase, and INH-NADH adduct formation activities." Protein Sci.; 19(3): 458-74.

[7] Cheng T, Li Q, Zhou Z, Wang Y, and Bryant, S.H (2012) "Structure-based virtual screening for drug discovery: A problem-centric review." AAPS J. 14, 133-141.

[8] Cole S.T, Brosch R, Parkhill J, et al. (June 1998) "Deciphering the biology of Mycobacterium tuberculosis from the complete genome sequence". Nature 393 (6685): 537-44.

[9] Davies Peter DO (1999) Multi-Drug Resistant Tuberculosis. Director of the Tuberculosis Research Unit, Cardiothoracic Centre, Thomas Drive, Liverpool.

[10] Dominguez C, Boelens R, and Bonvin A.M.JJ. Haddock (2003) "A protein-protein docking approach based on biochemical or biophysical information." J. Am. Chem. Soc.; 125:1731-1737.

[11] Gyanu Lamichhane. Novel targets in M. tuberculosis: search for new drugs. Trends in Molecular Medicine, Volume 17, Issue 1; 25-33, 2011.

[12] Ismael Kassim, Ray CG (editors) (2004) Sherris Medical Microbiology (4th Ed.). McGraw Hill.

[13] Junie B. Billones, Maria Constancia O. Carrillo, Voltaire G. Organi, Stephani Joy Y.Macalino, Inno A. Emnacen and Jamie Bernadette A. Sy. Virtual Screening against Mycobacterium tuberculosis Lipoate Protein Ligase B (MtbLipB) and In Silico ADMET Evaluation of Top Hits. Orient. J. Chem., Vol. 29(4); 1457-1468, 2013. 
[14] Kastritis P.L, Bonvin A. M. (2012). "On the binding affinity of macromolecular interactions: daring to ask why proteins interact." J R Soc Interface 10(79).

[15] Kitchen D.B, Decornez H, Furr J.R, Bajorath J (2004) "Docking and scoring in virtual screening for drug discovery: methods and applications". Nature reviews. Drug discovery 3 (11): 935-949.

[16] "Protein Docking Using Spherical Polar Fourier Correlations" Hex 8.0.0 User Manual.

[17] Ma Q, Zhao X, Nasser Eddine A, Geerlof A, Li X, Cronan JE, Kaufmann S.H, Wilmanns M (2006) "The Mycobacterium tuberculosis LipB enzyme functions as a cysteinelysine dyad acyltransferase" PNAS, vol. 103, no.23, 8662-8667.

[18] Pereira de Jésus-Tran K, Côté P.L, Cantin L, Blanchet J, Labrie F, Breton R (2006). "Comparison of crystal structures of human androgen receptor ligand-binding domain complexed with various agonists reveals molecular determinants responsible for binding affinity." Protein Science, 15(5): 987-999.

[19] Quin H. Christensen and John E. Cronan (2010) "Lipoic Acid Synthesis: A New Family of Octanoyltransferases Generally Annotated as Lipoate Protein Ligases" Biochemistry, 49 (46), pp 10024-10036.

[20] Ritchie, D.W. (2003) Evaluation of protein docking predictions using Hex 3.1 in CAPRI rounds 1 and 2. Proteins, 52:98-106.

[21] Smith, G.R., Sternberg, M.J. (2002) "Prediction of proteinprotein interactions by docking methods." Curr. Opin. Struct. Biol., 12:28-35.

[22] Suarez J, Ranguelova K, Jarzecki A.A, Manzerova J, Krymov V, Zhao X, Yu S, Metlitsky L, Gerfen G.J, Magliozzo R.S. (2009). "An oxyferrous heme/protein-based radical intermediate is catalytically competent in the catalase reaction of Mycobacterium tuberculosis catalase-peroxidase (KatG)". The Journal of Biological Chemistry 284 (11): 7017 7029 .

[23] Taylor, J.S. and Burnett, R.M. DARWIN (2000) "A program for docking flexible molecules.” Proteins 41, 173-191.

[24] World Health Organization (2010) WHO endorses new rapid tuberculosis test.

[25] Zhao X, Miller J. R, and Cronan, J. E. (2005) "The reaction of LipB, the octanoyl-[acyl carrier protein]: protein Noctanoyltransferase of lipoic acid synthesis, proceeds through an acyl-enzyme intermediate" Biochemistry 44, 16737-46. 